\title{
A De Novo Cause of PGM3 Deficiency Treated with Hematopoietic Stem Cell Transplantation
}

\author{
Andrew Winslow ${ }^{1}\left[\right.$ [ Elizabeth R. Jalazo ${ }^{2} \cdot$ April Evans $^{3} \cdot$ Mike Winstead $^{3} \cdot \operatorname{Timothy~Moran~}^{1}$
}

Received: 1 September 2021 / Accepted: 7 December 2021 / Published online: 18 January 2022

(c) The Author(s), under exclusive licence to Springer Science+Business Media, LLC, part of Springer Nature 2021

To the Editor,

Phosphoglucomutase 3 (PGM3) is a glycosylation enzyme that catalyzes Glc-NAc-6-P conversion to Glc-NAc-1-P; its downstream product, UDP-GlcNAc, is an essential glycosylation precursor [1]. PGM3 deficiency is an autosomal recessive congenital disorder of glycosylation for which a variety of clinical features have been described: atopy, recurrent infections (especially cutaneous, respiratory, and gastrointestinal infections from Staphylococcus aureus, candidiasis, or viral etiologies), enteropathy, neurologic manifestations, bone marrow failure, and skeletal dysplasia [1-3]. PGM3 deficiency was first described in 2014 in patients with hyper-IgE syndrome (HIES) phenotype characterized by recurrent infections, atopy, and elevated serum $\operatorname{IgE}[1,2]$. Patients may also present during infancy with severe combined immunodeficiency (SCID), neutropenia, and skeletal defects. Symptomatic management of PGM3 deficiency may involve antimicrobial therapy and immunoglobulin replacement as applicable. Correction of immune system abnormalities by hematopoietic stem cell transplantation (HSCT) has been reported in some cases [3], but data on the efficacy and safety of HSCT for PGM3-deficient patients remains scarce.

We report the case of an infant with PGM3 deficiency due to compound heterozygous mutations in $P G M 3$, including a de novo missense variant, who presented with combined

Andrew Winslow

andrew.winslow@unc.edu

1 Department of Pediatric Allergy and Immunology, University of North Carolina School of Medicine, 030 MacNider Hall, CB 7231, Chapel Hill, NC 27599-7231, USA

2 Department of Genetics and Metabolism, University of North Carolina School of Medicine, Chapel Hill, NC, USA

3 Department of Pediatric Hematology/Oncology, University of North Carolina School of Medicine, Chapel Hill, NC, USA immunodeficiency and was treated with HSCT. At presentation the patient was a term 3460-g Hispanic female born to a 38-year-old multiparous HIV-negative mother via vaginal delivery following an uncomplicated gestation. There was no history of parental consanguinity or family history of known inborn errors of immunity, fetal demise, or early childhood death. Newborn screening collected at 24 h showed abnormal T-cell receptor excision circle (TREC) assay. Examination at 2 weeks of life was remarkable for oral and diaper candidiasis, with no obvious dysmorphic features. CBC with differential was remarkable for leukopenia $(3700$ cells $/ \mu \mathrm{L})$, lymphopenia (1900 cells/ $\mu \mathrm{L}$ ), severe neutropenia (300 cells/ $\mu \mathrm{L}$ ), and eosinophilia (900 cells $/ \mu \mathrm{L}$ ). Lymphocyte subset analysis revealed markedly decreased numbers of $\mathrm{CD}^{+}$ lymphocytes (305 cells/ $\mu \mathrm{L}$, normal $1928-5622$ cells $/ \mu \mathrm{L}$ ), $\mathrm{CD}^{+}{ }^{+} \mathrm{T}$ cells $(211$ cells $/ \mu \mathrm{L}$, normal $1199-4100$ cells $/ \mu \mathrm{L})$, and $\mathrm{CD}^{+}$T cells (47 cells $/ \mu \mathrm{L}$, normal $578-1598$ cells $/ \mu \mathrm{L}$ ). CD $19^{+}$B cells were also decreased $(398$ cells $/ \mu \mathrm{L}$, normal 636-2829 cells/ $\mu \mathrm{L})$, whereas the $\mathrm{CD} 16^{+} / \mathrm{CD}^{2} 6^{+}$natural killer cell count was slightly elevated $(1594$ cells $/ \mu \mathrm{L}$, normal 247-1427 cells $/ \mu \mathrm{L})$. Immunophenotyping of T cells revealed a low percentage of $\mathrm{CD} 4{ }^{+} \mathrm{CD} 45 \mathrm{RA}^{+}$naïve $\mathrm{T}$ cells $(18 \%$, normal 64-95\%) with a reciprocal increased percentage of $\mathrm{CD}^{+}{ }^{+} \mathrm{CD} 45 \mathrm{RO}^{+}$memory $\mathrm{T}$ cells $(81 \%$, normal $2-22 \%)$. Mitogen studies were notable for decreased $\mathrm{CD} 45^{+}$total lymphocyte proliferative response $(28.1 \%$, normal $\geq 49.9 \%)$ and decreased $\mathrm{CD}^{+} \mathrm{T}$ cell proliferative response $(39.6 \%$, normal $\geq 58.5 \%$ ) to phytohemagglutinin. Serum IgG level was normal for age $(895 \mathrm{mg} / \mathrm{dL}$, presumably via maternal transplacental transfer), but serum IgA and IgM levels were below the limit of detection $(<8 \mathrm{mg} / \mathrm{dL}$ and $<25 \mathrm{mg}$ / $\mathrm{dL}$, respectively). Serum $\mathrm{IgE}$ levels were normal for age at 12.4 IU/mL (normal 2-34 IU/mL). No significant abnormalities were detected on skeletal survey, liver ultrasound, kidney ultrasound, echocardiogram, or brain MRI. Interestingly, chest X-ray confirmed the presence of a thymic shadow. Given concern for a combined immunodeficiency, the patient was started on antimicrobial prophylaxis and 
immunoglobulin replacement. Filgrastim was initiated for neutropenia, and the patient demonstrated a partial response, with a median neutrophil count of 750 cells $/ \mu \mathrm{L}$.

On day of life 18, a targeted gene panel was performed which was negative for 25 disease-causing genes, accounting for approximately $90 \%$ of cases of SCID. Due to the high suspicion for a combined immunodeficiency, rapid wholegenome sequencing was performed at day of life 39 on an Illumina next-generation sequencing platform by PerkinElmer Genomics. The patient was found to be heterozygous for two pathogenic variants in the PGM3 gene: a $P G M 3$ c.1049 $\mathrm{T}>6$ (p.Ile350Thr) missense variant in the sugarbinding domain and a $P G M 3$ c. $1558 \mathrm{C}>\mathrm{T}$ (p.Arg520Ter) nonsense variant in the phosphate-binding domain. The p.Ile350Thr variant, which was maternally inherited, has been previously described as a hypomorphic mutation that decreases PGM3 enzymatic activity [4]. The p.Arg520Ter nonsense variant had been observed in seven alleles in the general population and was predicted to be pathogenic, but the variant had not been previously reported in patients with PGM3 deficiency or primary immunodeficiency. Interestingly, the p.Arg520Ter variant was not detected in either parent. Paternity was confirmed through comparison of single nucleotide polymorphisms, indicating that the p.Arg520Ter variant arose de novo.

Given the patient's cellular immunodeficiency and severe neutropenia, allogeneic HSCT was deemed potentially life-saving and was performed on day of life 105 using 5/6 HLA-matched umbilical cord blood. The patient underwent a reduced-intensity conditioning with alemtuzumab $(0.2 \mathrm{mg} / \mathrm{kg}$ daily from day -14 to day -10$)$, fludarabine $(1 \mathrm{mg} / \mathrm{kg}$ daily from day -8 to day -4$)$, and melphalan
$(4.7 \mathrm{mg} / \mathrm{kg}$ on day -3$)$. GVHD prophylaxis consisted of tacrolimus and mycophenolate mofetil. Her course was complicated by sinusoidal obstructive syndrome at HSCT day +7 , which resolved with defibrotide and diuretics. Neutrophil engraftment occurred on day +14 , and she has been independent of immunoglobulin replacement since day +102 . Her lymphocyte counts normalized by day +173 (Fig. 1). Mitogen studies at 6 and 12 months post-HSCT showed normal lymphocyte proliferative responses to phytohemagglutinin. The patient developed stage 2 acute GVHD of the skin (overall grade 1 acute GVHD) that responded to topical corticosteroids; tacrolimus was discontinued at 7 months post-HSCT. Chimerism studies show full donor engraftment with $>95 \%$ donor CD3 $+\mathrm{T}$ cells and CD33 + myeloid cells at 12 months post-HSCT. No additional HSCT complications or manifestations of PGM3 deficiency have been identified at 13 months posttransplant, and the patient has achieved normal growth and developmental milestones.

Our case demonstrates the importance of newborn screening and the need to maintain a high index of suspicion when evaluating for inborn errors of immunity. During the evaluation of this infant with abnormal TRECs, lymphopenia, neutropenia, and eosinophilia, we identified a previously unreported de novo mutation associated with PGM3 deficiency. While were unable to verify decreased PGM3 protein levels or functional activity due to the urgency for definitive treatment with HSCT and clinical research restrictions posed by the COVID-19 pandemic, the clinical phenotype and pathogenic mutations in PGM3 strongly support a diagnosis of PGM3 deficiency. As PGM3 deficiency was not originally identified with initial targeted genetic sequencing, we also
Fig. 1 a Schematic representation of PGM3 protein. Ile350Ter and Arg520Ter variants and protein subunits are depicted in red, while previously reported pathogenic PGM3 variants are depicted in black. b, c Absolute neutrophil count and lymphocyte subsets for our patient with PGM3 deficiency; black arrow indicates HSCT at day of life 105

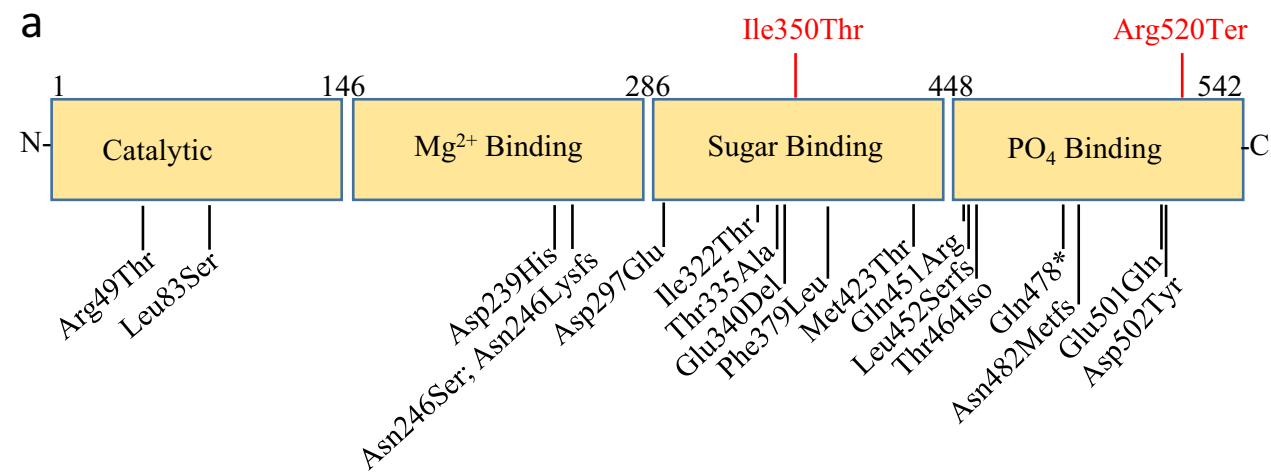

b

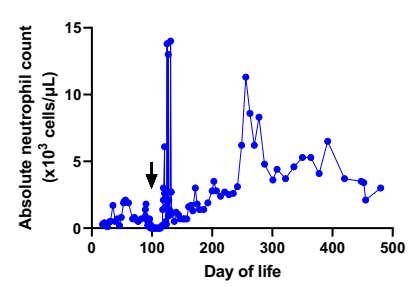

C

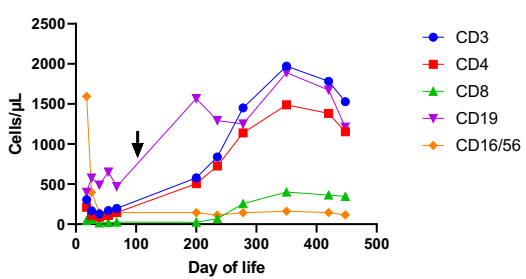


emphasize (1) the expanded role of large targeted sequencing panels in diagnosing inborn errors of immunity, which have a comparable cost profile to small targeted sequencing panels, and (2) that rapid whole-genome sequencing was critical in our infant's timely diagnosis.

The role of HSCT in the management of PGM3 deficiency has limited evidence to date. In 2014, Stray-Pedersen and colleagues described a patient with PGM3 deficiency who had HSCT at 4 months via 6/6 HLA-matched unrelated umbilical cord blood transplant, with correction of leukopenia and no infections, and neurodevelopmental status notable for global developmental delay [3]. A second patient also described by Stray-Pedersen and colleagues in 2014 had HSCT at 6 years via HLA-identical sibling umbilical cord blood, with resultant engraftment, correction of lymphocyte function, and neurodevelopmental status notable for mild speech delay [3]. Though these patients each had successful outcomes following transplantation, additional detail including conditioning regimen and long-term clinical outcomes are not available. In 2021, Fusaro and colleagues described a patient with PGM3 deficiency who had HSCT via HLA-matched sibling at 8 months of life following busulfan (cumulative area under the curve $21,984 \mu \mathrm{mol} / \mathrm{mL}$ ) and fludarabine $160 \mathrm{mg} / \mathrm{m}^{2}$ conditioning, resulting in full donor engraftment at day +14 . However, the patient had several postHSCT complications (pulmonary veno-occlusive disease, respiratory failure, autoimmune hemolytic anemia, chronic organizing pneumonia, neurologic impairment with hypotonia, and deafness) and eventually died at 8 months postHSCT from infection [5]. Our case illustrates that umbilical cord blood HSCT with reduced-intensity conditioning can safely and effectively correct immunodeficiency and neutropenia due to PGM3 deficiency, although the patient will require long-term surveillance for disease manifestations in other tissues.

This case also emphasizes the heterogeneous clinical phenotype of PGM3 deficiency. Our patient lacked the HIES-like features, skeletal abnormalities, and neurodevelopmental disabilities described in some patients with PGM3 deficiency [1,2], although this may be due in part to the early identification of our patient. Interestingly, individuals homozygous for the p.Ile350Thr missense variant identified in our patient were highly prone to infections but lacked abnormalities of the nervous or skeletal systems [4], suggesting that this mutation primarily affects immune function. The phenotypic consequences of the p.Arg520Ter nonsense variant in our patient are unclear, but other people with mutations in the phosphatebinding domain of PGM3 had severe T cell lymphopenia in the absence of dysmorphic findings or neurocognitive impairment [6]. Thus, it is likely that the p.Arg520Ter mutation in PGM3 also predominantly impacts immune cell function and/or development. As additional cases of PGM3 deficiency are identified as a result of newborn screening, we are eager to elucidate whether early treatment with HSCT corrects both immune and non-immunological manifestations of PGM3 deficiency.

Abbreviations Glc-NAc-6-P: N-acetyl-D-glucosamine-6-phosphate; Glc-NAc-1-P: N-acetyl-D-glucosamine-1-phosphate; UDP-GlcNAc: Uridine diphosphate N-acetylglucosamine; CBC: Complete blood count; MRI: Magnetic resonance imaging; GVHD: Graft versus host disease; PGM3: Phosphoglucomutase 3

Acknowledgements We thank Dr. Eric Weimer, Dr. Nathan Montgomery, and Thomas Felton for their respective roles in our patient's ongoing immunologic and genetic evaluation including genetic sequencing. We also acknowledge Cammie Presler, a nurse practitioner the bone marrow transplant unit, in providing outpatient follow-up.

Author Contribution Each author had substantial contributions in the completion of this manuscript, defined as follows. Andrew Winslow MD is the primary and corresponding author who composed and edited the draft and final versions of the publication. Elizabeth Jalazo MD is a co-author who reviewed the genetic section of the publication. April Evans MD is a co-author who reviewed the hematology/oncology section of the publication. Mike Winstead MD is a co-author who reviewed the hematology/oncology section of the publication and is the patient's primary bone marrow transplant clinician. Timothy Moran $\mathrm{MD} \mathrm{PhD} \mathrm{is} \mathrm{a} \mathrm{co-author} \mathrm{who} \mathrm{contributed} \mathrm{significantly} \mathrm{to} \mathrm{the} \mathrm{revisions}$ of the draft and final versions of the publication and is the patient's primary allergy/immunology clinician.

Data Availability Not applicable.

Code Availability Not applicable.

\section{Declarations}

Ethics Approval Not applicable.

Consent to Participate Not applicable.

Consent for Publication Not applicable.

Conflict of Interest The authors declare no competing interests.

\section{References}

1. Sassi A, et al. Hypomorphic homozygous mutations in phosphoglucomutase 3 (PGM3) impair immunity and increase serum IgE levels. J Allergy Clin Immunol. 2014;133(5):1410-9-1419.e1-13.

2. Zhang Y, et al. Autosomal recessive phosphoglucomutase 3 (PGM3) mutations link glycosylation defects to atopy, immune deficiency, autoimmunity, and neurocognitive impairment. J Allergy Clin Immunol. 2014;133(5):1400-9-1409.e1-5.

3. Stray-Pedersen A, et al. PGM3 mutations cause a congenital disorder of glycosylation with severe immunodeficiency and skeletal dysplasia. Am J Hum Genet. 2014;95(1):96-107.

4. Lundin KE, et al. Susceptibility to infections, without concomitant hyper-IgE, reported in 1976, is caused by hypomorphic mutation 
in the phosphoglucomutase 3 (PGM3) gene. Clin Immunol. 2015;161(2):366-72.

5. Fusaro M, et al. Two novel homozygous mutations in phosphoglucomutase 3 leading to severe combined immunodeficiency, skeletal dysplasia, and malformations. J Clin Immunol. 2021;41(5):958-66.
6. García-García A, et al. Novel PGM3 compound heterozygous variants with IgE-related dermatitis, lymphopenia, without syndromic features. Pediatr Allergy Immunol. 2021;32(3):566-75.

Publisher's Note Springer Nature remains neutral with regard to jurisdictional claims in published maps and institutional affiliations. 